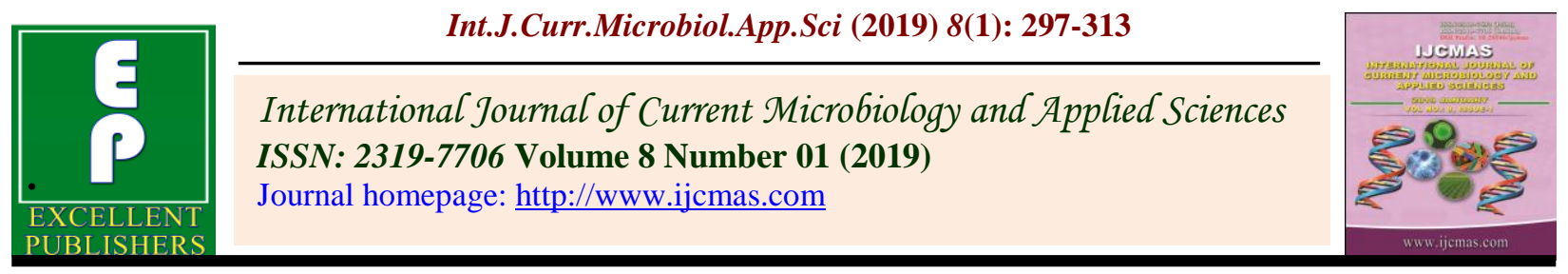

Original Research Article

https://doi.org/10.20546/ijcmas.2019.801.032

\title{
Management of Cucumber Mosaic Virus (CMV) Disease in Chilli through Biotic Defense Inducers
}

\author{
V.V. Kavyashri* and N. Nagaraju \\ Department of Plant Pathology, College of Agriculture, UAS, GKVK, \\ Bengaluru-560 065, India \\ *Corresponding author
}

\begin{abstract}
A B S T R A C T
Keywords

Chilli, CMV, DI,

AUDPC, Defense

inducers

Article Info

Accepted:

04 December 2018

Available Online:

10 January 2019

Different biotic defense inducers such as, red seaweed extract (Kappaphycus alvarezii), plant extracts (Mirabilis jalapa L. and Bougainvillea spectabilis Willd.), biocontrol agents (Trichoderma harzianum and Pseudomonas fluorescens) and other defense inducing biomolecules viz., synthetic nucleoside, chitosan and virex-H were tested for their efficacy on Cucumber mosaic virus (CMV) infection in Chilli var. Pusa Jwala during Kharif201617and Kharif 2017-18under field conditions. Results showed that a significantly less PDI (35.29 \%) and AUDPC (1238.33) was observed in plants treated with Chitosan (0.1\%) with enhanced plant height $(49.19 \mathrm{~cm})$, number of branches $(19.52 \mathrm{no}$.), number of fruits per plant (187.87 no.), individual fruit weight $(2.44 \mathrm{~g})$, fresh fruit yield (483.56 g.plant $\left.{ }^{-1}\right)$, fresh weight $\left(4.59 \mathrm{t}^{-h^{-1}}\right)$, dry weight $\left(1.38 \mathrm{t}^{-h^{-1}}\right)$. Significant differences among the treatments by reducing the CMV disease with increased growth and yield was observed and is found superior over the untreated control.

\section{Introduction}

Chilli (Capsicum annuum L.) is an important spice grown for its fruits, which are used in green as well as ripe dried form for its pungency. Chilli belongs to the genus Capsicum, family Solanaceae. It has originated in Mexico, Southern Peru and Bolivia (Villalon, 1981). India is the largest producer of chilli in the world with a production of 1492.14 MT. The average national productivity of chilli in India is 1.92MT/ha (IHD, 2015). Major chilli growing states in India are Andhra Pradesh, Maharashtra, Karnataka and Tamil Nadu

which together contribute about 75 per cent of the total area (Bhadramurthy et al., 2009).

Chilli is attacked by more than 30 different viruses throughout the world and the estimated collective yield losses ranging from 15 to 50 per cent (Agranovsky, 1993; Green and Kim, 1991). CMV is found to be the most bounteous virus on chilli, especially in tropic and semi-tropic regions, which provide ideal conditions for the virus perpetuation and their vectors (Biswas et al., 2013; Myti et al., 2014). Infection of CMV in chilli often leads to losses of 10-20 per cent in yield and even if harvested. In severe infections, yield losses
\end{abstract}


may reach up to 60 per cent if plants are infected at an early stage (Ong et al., 1979), with the plants producing none or only very few fruits, which are small in size (Jones et al., 1991).

CMV exhibit complex symptoms viz., mosaic, mottle, leaf distortion, vein chlorosis and stunting (Plate 1) causing considerable loss in yield and plant vigor (Rashid et al., 2007). CMV is easily transmitted by mechanical inoculation as well as by more than 80 species of aphids in non-persistent manner (Palukaitis and Garcia-Arenal, 2003). The coat protein (CP) of CMV is a primary determinant of aphid transmission (Chen and Francki, 1990). Seed transmission of CMV has been accounted for 0 to 100 per cent in different host species, including weed species (Neergaard, 1977). Weed hosts function as a repository for the virus and serve as primary source of inoculum for the development of disease epidemics (Grube et al., 2000).

In order to conquer production losses, different approaches are generally been adopted for the management of plant viral diseases. Use of inducible defense mechanisms against viruses has been shown to be effective in many plant species (Murphy, 2006). Control of virus disease is only can be done by controlling its vector using insecticides. Besides not being effective, it also has negative impact on human health and environment. Incorrect use of insecticides in both types and doses often causes problems because they can increase production costs and can leave residuals on production (Astutiet al., 2013). Utilization of nonhazardous materials such as the use of plants extracts, seaweed extracts and bio-control agents and their effectiveness are being investigated. Several researcher reports indicated, plant extracts and other defense inducers could be used to control various causes of plant diseases and are able to induce plant resistance (Madhusudhan et al., 2005). Therefore, the present study was conducted to evaluate a few biotic inducers to induce systemic resistance and safe means of controlling virus infection in chilli under open field conditions.

\section{Materials and Methods}

A field experiment was carried out during two growing Kharif seasons of 2016-17 and 201718, at MRS, Hebbal, Bengaluru in order to investigate the impact of foliar application of various biotic inducers on CMV incidence, growth and yield of chilli in var. PusaJwala. The chilli seedlings were primed with different biotic inducers separately and planted in the field with a spacing of $60 \times 45$ $\mathrm{cm}$. Cultural practices, pest and disease were taken care of by following package of practices of UAS, GKVK, Bengaluru.

\section{Biotic inducers used for management of CMV disease}

The experiment was carried out by using different treatments such as, red seaweed extract (Kappaphycus alvarezii), plant extracts, biocontrol agents and other defence inducing bio-molecules (Table 1 and Plate 1).

\section{Preparation of crude extracts of botanicals}

Crude extracts were prepared from the leaves of $M$. jalapa L. (Sanjemallige) and $B$. spectabilis Willd. (Paper flower). Hundred grams of leaves was blended in a grinder containing $100 \mathrm{~mL}$ sterilized distilled water at a ratio of 1:1 (weight/volume). The sample was spun at low speed for 10-15 min., the blended material was squeezed through a sterile muslin cloth to get a crude liquid extract and it was filtered through Whatman No. 1 filter paper. The filtrate was collected in sterilized glass tubes and the tubes were sealed under aseptic condition. The water extract so 
obtained was used within 6 hours of preparation (Thippeswamy, 2010).

\section{Culture filtrates of biological control agents}

Culture filtrates of $T$. harzianum and $P$. fluorescens was obtained by growing on potato dextrose broth (PDB) and nutrient broth (NB). Conical flasks of half litre capacity containing $250 \mathrm{~mL} \mathrm{PDB}$ and $\mathrm{NB}$ were inoculated with $5 \mathrm{~mm}$ mycelial plugs of 7 days old culture grown on PDA and loopful culture of bacteria grown on nutrient agar respectively. Culture flasks were placed on an orbital shaker (150 rotations. $\mathrm{min}^{-1}$ ) during the first week. After inoculation, culture flasks were checked visually and flasks showing only pure growth of their respective isolate after 35days of incubation were taken to collect culture filtrates. The liquid medium was filtered through oven dried Whatman No.42 filter paper to separate fungal mycelium. Filtrate containing cell suspension was collected and was considered as 100per cent concentration (Vishwanath and Kolte, 1997).

\section{Evaluation of biotic defense inducers}

Foliar application of biotic inducers was done at 15 days intervals starting from a week after transplanting in the field. The observations for disease incidence and growth parameters were recorded at before initiation of first spray and 15 days after each spray and the data were analyzed statistically. The per cent disease inhibition over control was calculated by using the formula given by Vincent (1927).

$$
\text { Disease inhibition }(\%)=\frac{(\mathrm{C}-\mathrm{T})}{\mathrm{C}}
$$

Where,

$\mathrm{C}=$ Per cent disease in untreated control

$\mathrm{T}=$ Per cent disease in treatment
Per cent yield increase over untreated $=$

$$
\text { Yt - Yc }
$$

Where, Yt- Yield of treated plant

Yc- yield of untreated control plant

Area under disease progress curve (AUDPC)

The Area under disease progressive curve was calculated by the trapezoidal integration of the percent disease index (PDI) over time for each treatmentaccording to Campbell and Madden (1990).

AUDPC $=\sum_{\mathrm{i}=1}^{\mathrm{n}-1} \frac{\left(\mathrm{y}_{\mathrm{i}}+\mathrm{y}_{\mathrm{i}}+1\right)}{2} \times\left(\mathrm{t}_{\mathrm{i}}+1-\mathrm{t}_{\mathrm{i}}\right)$

where,

$\mathrm{n}$ : Number of assessment;

$\mathrm{y}$ : Percent disease index (PDI)

$\left(t_{i+1}-t_{i}\right)$ is duration between two consecutive assessments

The disease assessments over specific periods of time interval viz., 30, 45 and 60 DAS was recorded during the experiments and interpreted according to the above mentioned formula. The AUDPC of CMV for each treatment was calculated and the data was analysed statistically.

\section{Statistical analysis}

All treatments were replicated thrice and organized in a randomized complete block design (RCBD). Data acquired from the present investigation was subjected to ANOVA (Sheoran et al., 1998). Significance between the treatments were calculated according to Duncan's Multiple Range Test at $\mathrm{p}=0.05$. 


\section{Results and Discussion}

\section{Effect of different treatments on systemic protection against CMV in chilli}

In the present study, an attempt has been made to control CMV by using certain biotic defense inducers during Kharif201617andKharif2017-18 under field conditions (Plate 2). The antiviral activity of different biotic inducers was assessed for their effect based on PDI and AUDPC. Plants exhibited different kind of symptoms includes mosaic, leaf distortion, rat tailling, and dwarfing (Plate 3) under field conditions.

At 65 DAT, chilli plants treated with chitosan (0.1\%) showed less PDI of 29.83 and 36.64 per cent respectively in Kharif2016-17 and Kharif 2017-18, followed by K. alvarezii (30.35 and $39.48 \%$ ) and $P$. fluorescens $(31.10$ and $41.04 \%)$ compared to control (49.07 and $62.06 \%$ ) (Table 2, Fig. 1). In addition, PDI was directly correlated with the AUDPC, chitosan treated plants showed AUDPC of 1162.35 and 1314.30 followed by K. alvarezii (1098.68 and 1425.55), P. fluorescens (1133.33 and 1584.28). Whereas, untreated control showed high AUDPC of 1768.84 and 2216.79 in Kharif 2016-17and Kharif 201718. The present investigations indicated that, there was a significant reduction in the severity of CMV disease in chilli treated with different biotic inducers compared to that of the untreated control.

The effectiveness of chitosan application for viral disease management under field condition has been reported by several workers (Hadrami et al., 2010; Compant et al., 2010; Noiket et al., 2014). Chitosan treatment caused inhibition of tomato yellow leaf curl disease symptoms in tomato (Noiket et al., 2014). The field experiments conducted for the management of CMV in gherkins using seaweed extracts during Kharif and rabi 2016 revealed that $K$. alvarezii- $1(0.4 \%)$ recorded less mean PDI of 16.65 and 16.06 followed by Halymenia durvillae (1\%) with mean PDI (18.34 and 18.98) compared to control with mean PDI (31.77 and 31.96) respectively (Venkatesh, 2016). Pushpa et al., (2018) reported the delay in appearance of PRSV symptoms in papaya plants treated with $K$. alvarezii $(0.4 \%)$ and produced more number of fruits (average 30 no's per plant) compared to untreated control (average 15 no's per plant). Similarly, biocontrol efficacy of $P$. fluorescens against CMV, tomato mottle virus and tomato spotted wilt virus (TSWV) in tomato under field conditions was observed by Murphy et al., (2000) and Kandan et al., (2003).

Chitosan induced resistance is may be due to increased activity chitinase enzyme, chitosan may inhibit virus replication, cell-to-cell movement and also activate receptor like kinases (RLK's) which induce systemic resistance (ISR) in the plants (DeniFirmansyah et al., 2017). The increased chitinase activity might have been prevented the damage caused by viral pathogen and, thus, increased the per cent disease control in chitosan treated plants. Synthesis and accumulation of PR proteins have been reported to play an important role in plant defence mechanisms. Chitinases, which are classified under PR-3 have been reported to associate with resistance in plants against pests and diseases (Maurhofer et al., 1994; Van-Loon, 1997).

The PGPR, a biocontrol agent induce ISR by activating jasmonate and ethylene signalling pathways systemically in plants and these hormones stimulate the defence responses in host plants against a variety of plant pathogens (Deni-Firmansyah et al., 2017). Rhizobacterial treated tomato plants showed to induce phenylalanine ammonia lyase (PAL), peroxidase $(\mathrm{PO})$, Chitinase and polyphenol 
oxidase (PPO). PAL activity during plant pathogen and plant - pest interactions (Harish, 2005) and is known to play an important role in the biosynthesis of various defense chemicals in phenyl propanoid metabolism (Daayf et al., 1997). Cinammic acid, the product of PAL, is directly linked to cell lignifications processes and the highest levels of PAL activity usually occur about one day after initial infection of pathogen (Podile and Laxmi, 1998).

The molecular details on the effect of $K$. alvarezii extract in delaying CMV symptoms remain to be explored. However, it is likely that the sulphated galactans of $K$. alvarezii may activate plant's immune responses similarly as microbial elicitors viz., bacterial peptidoglycans, flagellin, lipopolysaccharides and chitin of fungal cell wall that elicit MAMPs immune response (Boller and Felix, 2009; Macho and Zipfel, 2014). More recently, the red seaweed Schyzimenia binderi derived oligo-sulphated-galactan, poly-Ga, has been shown to induce long-term protection against TMV in tobacco plants (Vera et al.,
2011). It is possible that the sulphated oligosugars or any unknown chemical compounds present in $K$. alvarezii extract may mimic as MAMP elicitors leading to activation of plant's immune response, however further studies in this direction are essential for detailed understanding of their molecular mechanisms.

\section{Plant growth promotional potential of different biotic inducers}

The effect of different biotic inducers on growth and yield parameters of chilli was studied under field conditions. Chilli plants treated with $P$. fluorescens $(0.6 \%)$ showed significantly increased plant height $(51.42 \mathrm{~cm})$, number of branches (22.36) and less number of days for initial flowering (32.12) in addition to suppression of CMV disease, followed by chitosan $(0.1 \%)$ and $K$. alvarezii $(0.4 \%)$ (Table 3, Fig. 2).However compared to other treatments, treatments viz., Virex-H and extract of Mirabilis jalapa L. (sanjemallige) resulted in significantly reduced growth.

Table.1 List of treatments used against CMV disease in chilli

\begin{tabular}{|l|l|c|}
\hline \multicolumn{1}{|c|}{ Treatments } & $\begin{array}{c}\text { Dosage } \\
(\%)\end{array}$ \\
\hline T1 & $\begin{array}{l}\text { Red seaweed (Kappaphycus alvarezii (Doty) Doty ex } \\
\text { P.C.Silva) extract }\end{array}$ & 0.4 \\
\hline T2 & Synthetic nucleoside & 0.25 \\
\hline T3 & Extract of Mirabilis jalapa L. (Sanjemallige) & 0.5 \\
\hline T4 & Extract of Bougainvillea spectabilisWilld. (Paper flower) & 0.5 \\
\hline T5 & Fungal bio-control agent (Trichoderma harzianum) & 0.6 \\
\hline T6 & Bacterial bio-control agent (Pseudomonas fluorescens) & 0.6 \\
\hline T7 & Chitosan & 0.1 \\
\hline T8 & Virex-H & 0.3 \\
\hline T9 & Dimethoate 30per cent EC & 0.2 \\
\hline T10 & Untreated control & \\
\hline
\end{tabular}


Table.2 Effect of biotic inducers on incidence of CMV disease during Kharif-2016-17 and 2017-18 under field conditions

\begin{tabular}{|c|c|c|c|c|c|c|c|c|c|c|c|}
\hline \multirow{2}{*}{\multicolumn{2}{|c|}{ Treatments }} & \multirow{2}{*}{\multicolumn{5}{|c|}{ Kharif- 2016-17 }} & \multicolumn{5}{|c|}{ Kharif- 2017-18 } \\
\hline & & & & & & & & & & & \\
\hline & & \multirow{2}{*}{$\begin{array}{c}\text { PDI@ } \\
30 \\
\text { DAT } \\
21.30^{\mathrm{de}}\end{array}$} & \multirow{2}{*}{$\begin{array}{c}\text { PDI } \\
\text { @ } 45 \\
\text { DAT } \\
26.12^{\text {gf }}\end{array}$} & \multirow{2}{*}{$\begin{array}{c}\text { PDI @ } \\
60 \\
\text { DAT } \\
30.35^{\mathrm{f}}\end{array}$} & \multirow{2}{*}{$\begin{array}{l}\text { AUDPC } \\
1098.68\end{array}$} & \multirow{2}{*}{$\begin{array}{c}\text { DOC } \\
(\%) @ \\
\text { 60 DAT } \\
38.15\end{array}$} & \multirow{2}{*}{$\begin{array}{c}\text { PDI @ } \\
\text { 30 } \\
\text { DAT } \\
28.62^{\mathrm{f}}\end{array}$} & \multirow{2}{*}{$\begin{array}{c}\text { PDI@ } \\
45 \\
\text { DAT } \\
32.36^{\mathrm{f}}\end{array}$} & \multirow{2}{*}{$\begin{array}{c}\text { PDI @ } \\
60 \\
\text { DAT } \\
39.48^{\mathrm{g}}\end{array}$} & \multirow{2}{*}{$\begin{array}{l}\text { AUDPC } \\
1425.55\end{array}$} & \multirow{2}{*}{$\begin{array}{c}\text { DOC } \\
(\%) @ \\
\text { 60 DAT } \\
36.38\end{array}$} \\
\hline T1 & $\begin{array}{l}K . \text { alvarezii (Doty) Doty ex } \\
\text { P.C.Silva) @ } 0.4 \%\end{array}$ & & & & & & & & & & \\
\hline $\mathbf{T} 2$ & Synthetic nucleoside @ 0.25\% & $23.83^{c}$ & $32.45^{\mathrm{d}}$ & $35.38^{d}$ & 1288.26 & 27.90 & $30.27^{e}$ & $41.29^{c}$ & $45.50^{\mathrm{cd}}$ & 1641.71 & 26.68 \\
\hline T3 & Mirabilis jalapa L.@0.5\% & $20.02^{\mathrm{e}}$ & $30.00^{\mathrm{e}}$ & $31.37^{\mathrm{e}}$ & 1135.60 & 36.07 & $27.61^{\mathrm{g}}$ & $41.45^{c}$ & $43.81^{\text {de }}$ & 1571.60 & 29.41 \\
\hline T4 & B. spectabilisWilld. @ 0.5\% & $21.32^{\mathrm{de}}$ & $26.37^{\mathrm{gf}}$ & $31.80^{\mathrm{e}}$ & 1113.67 & 35.19 & $30.59^{\mathrm{e}}$ & $37.89^{d}$ & $46.19^{c}$ & 1603.03 & 25.57 \\
\hline T5 & T.harzianum @0.6\% & $20.10^{\mathrm{e}}$ & $26.10^{\mathrm{gf}}$ & $31.49^{\mathrm{e}}$ & 1079.94 & 35.83 & $27.11^{\mathrm{g}}$ & $35.26^{\mathrm{e}}$ & $42.99^{\text {ef }}$ & 1461.13 & 30.73 \\
\hline T6 & P.fluorescens@0.6\% & $22.83^{\mathrm{cd}}$ & $25.77^{\mathrm{g}}$ & $31.10^{\mathrm{e}}$ & 1133.33 & 36.62 & $32.53^{d}$ & $36.31^{\mathrm{de}}$ & $41.04^{\mathrm{fg}}$ & 1584.28 & 33.87 \\
\hline $\mathbf{T 7}$ & Chitosan@0.1\% & $23.94^{\mathrm{c}}$ & $26.67^{f}$ & $29.83^{f}$ & 1162.35 & 39.21 & $25.40^{h}$ & $31.20^{f}$ & $36.64^{h}$ & 1314.30 & 40.96 \\
\hline T8 & Virex-H@0.3\% & $34.55^{\mathrm{a}}$ & $42.14^{b}$ & $47.80^{b}$ & 1767.87 & 2.59 & $37.55^{b}$ & $45.88^{b}$ & $52.60^{b}$ & 1927.58 & 15.24 \\
\hline T9 & Dimethoate 30\% EC @ 0.2\% & $30.02^{b}$ & $37.27^{\mathrm{c}}$ & $45.63^{c}$ & 1576.81 & 7.01 & $33.79^{c}$ & $42.02^{\mathrm{c}}$ & $51.99^{b}$ & 1780.45 & 16.23 \\
\hline T10 & Untreated control & $33.44^{\mathrm{a}}$ & $43.23^{\mathrm{a}}$ & $49.07^{\mathrm{a}}$ & 1768.84 & 0.00 & $41.77^{\mathrm{a}}$ & $54.10^{\mathrm{a}}$ & $62.06^{\mathrm{a}}$ & 2216.79 & 0.00 \\
\hline \multicolumn{2}{|c|}{ SEm \pm} & 0.498 & 0.146 & 0.176 & -- & -- & 0.233 & 0.465 & 0.520 & -- & -- \\
\hline \multicolumn{2}{|c|}{ CD@ $9 \%$} & 1.479 & 0.435 & 0.522 & -- & -- & 0.693 & 1.383 & 1.544 & -- & -- \\
\hline \multicolumn{2}{|c|}{ CV \% } & 3.430 & 0.801 & 0.836 & -- & -- & 1.281 & 2.027 & 1.947 & -- & -- \\
\hline
\end{tabular}

*DOS: 20-08-2017; DOT: 28-09-2017

PDI: Per cent disease incidence; DAT: Days after transplanting; AUDPC: Area under disease progress curve; DOC: Decrease over control; In each column, means followed by the same letter are not significantly different at $\mathrm{p}=0.05$ according to Duncan's Multiple Range Test. 
Table.3 Effect of biotic inducers on growth parameters of chilli during Kharif 2016-17 and 2017-18 under field conditions

\begin{tabular}{|c|c|c|c|c|c|c|c|}
\hline \multicolumn{2}{|r|}{ Treatments } & \multicolumn{3}{|c|}{ Kharif- 2016-17 } & \multicolumn{3}{|c|}{ Kharif- 2017-18 } \\
\hline & & \multirow{2}{*}{$\begin{array}{c}\begin{array}{c}\text { Plant } \\
\text { height } \\
(\mathbf{c m})\end{array} \\
46.50^{\mathrm{d}}\end{array}$} & \multirow{2}{*}{$\begin{array}{c}\begin{array}{c}\text { Number } \\
\text { of } \\
\text { branches }\end{array} \\
\text { (no.) } \\
18.75^{\mathrm{b}}\end{array}$} & \multirow{2}{*}{$\begin{array}{c}\text { Days to } \\
\text { initiation } \\
\text { of } \\
\text { flowering } \\
37.44^{\mathrm{g}}\end{array}$} & \multirow{2}{*}{$\begin{array}{c}\begin{array}{c}\text { Plant } \\
\text { height } \\
(\mathbf{c m})\end{array} \\
47.20^{\mathrm{c}}\end{array}$} & \multirow{2}{*}{$\begin{array}{c}\begin{array}{c}\text { Number } \\
\text { of } \\
\text { branches } \\
\text { (no.) }\end{array} \\
19.40^{\mathrm{b}}\end{array}$} & \multirow{2}{*}{$\begin{array}{c}\begin{array}{c}\text { Days to } \\
\text { initiation } \\
\text { of }\end{array} \\
\text { flowering } \\
37.86^{\mathrm{g}}\end{array}$} \\
\hline T1 & K. alvarezii (Doty) Doty ex P.C.Silva) @ 0.4\% & & & & & & \\
\hline $\mathbf{T} 2$ & Synthetic nucleoside@ $0.25 \%$ & $45.52^{\mathrm{e}}$ & $12.54^{\mathrm{d}}$ & $40.56^{\mathrm{e}}$ & $46.13^{d}$ & $12.96^{\mathrm{d}}$ & $41.19^{\mathrm{e}}$ \\
\hline T3 & Mirabilis jalapa L.@ 0.5\% & $40.77^{\mathrm{g}}$ & $9.56^{f}$ & $43.35^{\mathrm{c}}$ & $41.24^{\mathrm{f}}$ & $10.23^{\mathrm{f}}$ & $43.85^{c}$ \\
\hline T4 & Bougainvillea spectabilisWilld. @ 0.5\% & $42.36^{\mathrm{f}}$ & $11.45^{\mathrm{e}}$ & $42.00^{d}$ & $42.89^{\mathrm{e}}$ & $11.36^{\mathrm{e}}$ & $42.15^{\mathrm{d}}$ \\
\hline T5 & Trichodermaharzianum @ 0.6\% & $48.56^{\mathrm{c}}$ & $16.35^{\mathrm{c}}$ & $39.54^{\mathrm{f}}$ & $49.23^{b}$ & $16.42^{\mathrm{c}}$ & $40.02^{f}$ \\
\hline T6 & Pseudomonas fluorescens @ 0.6\% & $51.42^{\mathrm{a}}$ & $22.36^{\mathrm{a}}$ & $32.12^{i}$ & $52.14^{\mathrm{a}}$ & $22.15^{\mathrm{a}}$ & $32.45^{i}$ \\
\hline T7 & Chitosan@0.1\% & $49.25^{b}$ & $19.24^{b}$ & $35.24^{h}$ & $49.13^{b}$ & $19.80^{b}$ & $35.36^{\mathrm{h}}$ \\
\hline T8 & Virex-H@0.3\% & $39.35^{h}$ & $8.90^{\mathrm{g}}$ & $45.23^{b}$ & $39.86^{\mathrm{g}}$ & $9.70^{\mathrm{gf}}$ & $45.75^{b}$ \\
\hline T9 & Dimethoate 30\% EC @ 0.2\% & $46.23^{d}$ & $12.75^{\mathrm{d}}$ & $40.25^{\mathrm{e}}$ & $46.53^{\text {cd }}$ & $13.40^{\mathrm{d}}$ & $40.90^{\mathrm{e}}$ \\
\hline T10 & Untreated control & $37.45^{i}$ & $8.65^{\mathrm{g}}$ & $46.30^{\mathrm{a}}$ & $36.70^{h}$ & $9.14^{\mathrm{g}}$ & $46.78^{a}$ \\
\hline \multicolumn{2}{|c|}{ SEm \pm} & 0.092 & 0.121 & 0.085 & 0.179 & 0.16 & 0.193 \\
\hline \multicolumn{2}{|c|}{ CD@ $5 \%$} & 0.273 & 0.359 & 0.253 & 0.532 & 0.476 & 0.574 \\
\hline \multicolumn{2}{|c|}{$\mathrm{CV} \%$} & 0.356 & 1.49 & 0.367 & 0.688 & 1.92 & 0.823 \\
\hline
\end{tabular}

*DOS: 20-08-2017; DOT: 28-09-2017; In each column, means followed by the same letter are not significantly different at $\mathrm{p}=0.05$ according to Duncan's Multiple Range Test. 
Table.4 Effect of biotic inducers on yield parameters of chilli during Kharif 2016-17 and 2017-18 under field conditions

\begin{tabular}{|c|c|c|c|c|c|c|c|c|c|c|c|c|}
\hline \multirow{2}{*}{$\begin{array}{c}\text { Treatment } \\
\text { S }\end{array}$} & \multicolumn{6}{|c|}{ Kharif- 2016-17 } & \multicolumn{6}{|c|}{ Kharif- 2017-18 } \\
\hline & $\begin{array}{c}\text { Number } \\
\text { of fruits } \\
\text { per } \\
\text { plant }\end{array}$ & $\begin{array}{l}\text { Individu } \\
\text { al fruit } \\
\text { weight } \\
\text { (g) }\end{array}$ & $\begin{array}{c}\text { Fresh } \\
\text { fruit } \\
\text { yield } \\
\text { (g/plant) }\end{array}$ & $\begin{array}{c}\text { Fresh } \\
\text { weight } \\
\left(\text { t.ha }^{-1}\right)\end{array}$ & $\begin{array}{c}\text { Dry } \\
\text { weight } \\
\left(\text { t.ha }^{-1}\right)\end{array}$ & $\begin{array}{l}\text { IOC } \\
(\%)\end{array}$ & $\begin{array}{c}\text { Number } \\
\text { of fruits } \\
\text { per } \\
\text { plant }\end{array}$ & $\begin{array}{l}\text { Individu } \\
\text { al fruit } \\
\text { weight } \\
\text { (g) }\end{array}$ & $\begin{array}{c}\text { Fresh } \\
\text { fruit yield } \\
\text { (g/plant) }\end{array}$ & $\begin{array}{c}\text { Fresh } \\
\text { weight } \\
\left(\text { t.ha }^{-1}\right)\end{array}$ & $\begin{array}{c}\text { Dry } \\
\text { weight } \\
\left(\text { t.ha }^{-1}\right)\end{array}$ & $\begin{array}{l}\text { IOC } \\
(\%)\end{array}$ \\
\hline T1 & $178.25^{b}$ & $2.48^{\mathrm{a}}$ & $475.41^{b}$ & $4.74^{a b}$ & $1.42^{\mathrm{ab}}$ & 16.67 & $169.35^{b}$ & $2.21^{\mathrm{ab}}$ & $459.23^{b}$ & $4.13^{\mathrm{ab}}$ & $1.24^{\mathrm{ab}}$ & 21.55 \\
\hline $\mathbf{T} 2$ & $159.53^{d}$ & $2.35^{\mathrm{a}}$ & $448.60^{d}$ & $4.57^{\mathrm{ab}}$ & $1.37^{\mathrm{ab}}$ & 13.57 & $150.03^{d}$ & $2.13^{\mathrm{ab}}$ & $434.20^{c}$ & $3.98^{a b c}$ & $1.19^{a b c}$ & 18.59 \\
\hline T3 & $122.98^{h}$ & $2.01^{\mathrm{a}}$ & $429.42^{h}$ & $4.02^{b}$ & $1.21^{\mathrm{b}}$ & 1.74 & $113.40^{h}$ & $1.83^{\mathrm{ab}}$ & $413.56^{h}$ & $3.41^{\mathrm{cd}}$ & $1.02^{\mathrm{cd}}$ & 4.99 \\
\hline T4 & $137.25^{\mathrm{g}}$ & $2.13^{a}$ & $431.21^{\mathrm{g}}$ & $4.23^{a b}$ & $1.27^{\mathrm{ab}}$ & 6.62 & $127.80^{\mathrm{g}}$ & $1.86^{\mathrm{ab}}$ & $415.80^{g}$ & $3.63^{\mathrm{bcd}}$ & $1.09^{\mathrm{bcd}}$ & 10.74 \\
\hline T5 & $142.50^{f}$ & $2.20^{\mathrm{a}}$ & $435.54^{f}$ & $4.29^{a b}$ & $1.29^{\mathrm{ab}}$ & 7.93 & $133.13^{f}$ & $1.93^{\mathrm{ab}}$ & $421.21^{f}$ & $3.70^{\mathrm{abcd}}$ & $1.11^{\mathrm{abcd}}$ & 12.43 \\
\hline T6 & $162.80^{c}$ & $2.42^{\mathrm{a}}$ & $456.24^{c}$ & $4.68^{a b}$ & $1.40^{\mathrm{ab}}$ & 15.60 & $152.54^{c}$ & $2.18^{a b}$ & $430.36^{d}$ & $3.97^{\mathrm{abc}}$ & $1.19^{\mathrm{abc}}$ & 18.39 \\
\hline $\mathbf{T 7}$ & $192.70^{\mathrm{a}}$ & $2.56^{\mathrm{a}}$ & $490.86^{\mathrm{a}}$ & $4.89^{\mathrm{a}}$ & $1.47^{\mathrm{a}}$ & 19.22 & $183.04^{\mathrm{a}}$ & $2.32^{\mathrm{a}}$ & $476.25^{a}$ & $4.29^{a}$ & $1.29^{\mathrm{a}}$ & 24.48 \\
\hline T8 & $120.20^{\mathrm{i}}$ & $1.98^{\mathrm{a}}$ & $426.12^{i}$ & $3.98^{b}$ & $1.19^{b}$ & 0.75 & $110.50^{i}$ & $1.73^{b}$ & $410.68^{i}$ & $3.38^{\mathrm{cd}}$ & $1.01^{\mathrm{cd}}$ & 4.14 \\
\hline T9 & $143.60^{\mathrm{e}}$ & $2.26^{\mathrm{a}}$ & $436.58^{e}$ & $4.36^{a b}$ & $1.31^{\mathrm{ab}}$ & 9.40 & $134.20^{\mathrm{e}}$ & $2.12^{\mathrm{ab}}$ & $422.34^{\mathrm{e}}$ & $3.77^{\mathrm{abcd}}$ & $1.13^{\mathrm{abcd}}$ & 14.06 \\
\hline T10 & $112.36^{\mathrm{j}}$ & $1.90^{\mathrm{a}}$ & $425.36^{j}$ & $3.95^{b}$ & $1.19^{b}$ & 0.00 & $103.40^{j}$ & $1.71^{b}$ & $409.53^{j}$ & $3.24^{d}$ & $0.97^{d}$ & 0.00 \\
\hline SEm \pm & 0.151 & 0.154 & 0.127 & 0.172 & 0.052 & -- & 0.141 & 0.115 & 0.110 & 0.144 & 0.043 & -- \\
\hline CD@ $9 \%$ & 0.447 & 0.457 & 0.377 & 0.511 & 0.153 & -- & 0.418 & 0.341 & 0.326 & 0.429 & 0.129 & -- \\
\hline CV \% & 0.177 & 11.949 & 0.049 & 6.811 & 6.811 & -- & 0.177 & 9.940 & 0.044 & 6.669 & 6.669 & -- \\
\hline
\end{tabular}

*DOS: 20-08-2017; DOT: 28-09-2017

IOC: Increase over control; In each column, means followed by the same letter are not significantly different at $\mathrm{p}=0.05$ according to Duncan's Multiple Range Test 
Table.5 Effect of biotic defense inducers on CMV disease incidence, growth and yield parameters of chilli under field conditions*

\begin{tabular}{|c|c|c|c|c|c|c|c|c|c|c|c|}
\hline Treatments & $\begin{array}{c}\text { PDI @ } \\
30 \\
\text { DAT }\end{array}$ & $\begin{array}{c}\text { PDI @ } \\
45 \text { DAT }\end{array}$ & $\begin{array}{l}\text { PDI @ } \\
60 \text { DAT }\end{array}$ & $\begin{array}{l}\text { Plant } \\
\text { height } \\
(\mathrm{cm})\end{array}$ & $\begin{array}{l}\text { Number } \\
\text { of } \\
\text { branches } \\
\text { (no.) }\end{array}$ & $\begin{array}{c}\text { Days to } \\
\text { initiation } \\
\text { of } \\
\text { flowering }\end{array}$ & $\begin{array}{c}\text { Number } \\
\text { of fruits } \\
\text { per } \\
\text { plant }\end{array}$ & $\begin{array}{l}\text { Individual } \\
\text { fruit } \\
\text { weight(g) }\end{array}$ & $\begin{array}{c}\text { Fresh } \\
\text { fruit yield }^{-1} \\
(\text { g.plant }\end{array}$ & $\begin{array}{c}\text { Fresh } \\
\text { weight } \\
\left(\text { t.ha }^{-1}\right)\end{array}$ & $\begin{array}{c}\text { Dry } \\
\text { weight } \\
\left(\text { t.ha }{ }^{-1}\right)\end{array}$ \\
\hline T1 & $23.35^{\mathrm{g}}$ & $28.66^{h}$ & $33.50^{h}$ & $46.85^{d}$ & $19.08^{c}$ & $37.65^{\mathrm{g}}$ & $173.80^{b}$ & $2.35^{\mathrm{ab}}$ & $467.32^{b}$ & $4.44^{\mathrm{ab}}$ & $1.33^{\mathrm{a}}$ \\
\hline $\mathbf{T} 2$ & $28.24^{d}$ & $31.49^{g f}$ & $35.44^{\mathrm{g}}$ & $45.83^{f}$ & $12.75^{f}$ & $40.88^{e}$ & $154.78^{d}$ & $2.24^{\mathrm{abc}}$ & $441.40^{d}$ & $4.28^{a b c}$ & $1.28^{\mathrm{ab}}$ \\
\hline T3 & $23.82^{\mathrm{g}}$ & $35.73^{e}$ & $37.59^{f}$ & $41.01^{h}$ & $9.90^{\mathrm{h}}$ & $43.60^{c}$ & $118.19^{h}$ & $1.92^{\mathrm{de}}$ & $421.49^{h}$ & $3.72^{\mathrm{de}}$ & $1.11^{\mathrm{de}}$ \\
\hline T4 & $25.96^{f}$ & $32.13^{f}$ & $39.00^{e}$ & $42.63^{g}$ & $11.41^{\mathrm{g}}$ & $42.08^{d}$ & $132.53^{\mathrm{g}}$ & $2.00^{\text {cde }}$ & $423.51^{\mathrm{g}}$ & $3.93^{\text {cde }}$ & $1.18^{\text {cde }}$ \\
\hline T5 & $23.61^{\mathrm{g}}$ & $30.68^{\mathrm{g}}$ & $37.24^{f}$ & $48.90^{c}$ & $16.39^{d}$ & $39.78^{f}$ & $137.82^{f}$ & $2.07^{\text {bcde }}$ & $428.38^{f}$ & $4.00^{\text {cde }}$ & $1.20^{\mathrm{bcd}}$ \\
\hline T6 & $27.05^{\mathrm{e}}$ & $36.87^{d}$ & $40.44^{d}$ & $51.78^{a}$ & $22.26^{\mathrm{a}}$ & $32.29^{i}$ & $157.67^{c}$ & $2.30^{\mathrm{ab}}$ & $443.30^{c}$ & $4.33^{a b c}$ & $1.30^{\mathrm{ab}}$ \\
\hline T7 & $25.73^{f}$ & $29.07^{\mathrm{h}}$ & $35.29^{g}$ & $49.19^{b}$ & $19.52^{b}$ & $35.30^{h}$ & $187.87^{\mathrm{a}}$ & $2.44^{\mathrm{a}}$ & $483.56^{\mathrm{a}}$ & $4.59^{\mathrm{a}}$ & $1.38^{\mathrm{a}}$ \\
\hline T8 & $36.05^{b}$ & $44.01^{b}$ & $50.20^{b}$ & $39.61^{\mathrm{i}}$ & $9.30^{\mathrm{i}}$ & $45.49^{b}$ & $115.35^{\mathrm{i}}$ & $1.86^{\mathrm{e}}$ & $418.40^{i}$ & $3.68^{\mathrm{de}}$ & $1.10^{\mathrm{de}}$ \\
\hline T9 & $31.91^{c}$ & $39.65^{c}$ & $48.81^{c}$ & $46.38^{e}$ & $13.08^{\mathrm{e}}$ & $40.58^{e}$ & $138.90^{\mathrm{e}}$ & $2.19^{\mathrm{abcd}}$ & $429.46^{\mathrm{e}}$ & $4.07^{b c d}$ & $1.22^{\mathrm{bc}}$ \\
\hline T10 & $37.61^{\mathrm{a}}$ & $48.67^{\mathrm{a}}$ & $55.57^{\mathrm{a}}$ & $37.08^{j}$ & $8.90^{j}$ & $46.54^{\mathrm{a}}$ & $107.88^{j}$ & $1.81^{\mathrm{e}}$ & $417.45^{j}$ & $3.60^{\mathrm{e}}$ & $1.08^{\mathrm{e}}$ \\
\hline SEm \pm & 0.546 & 0.686 & 0.764 & 0.137 & 0.119 & 0.103 & 0.126 & 0.086 & 0.779 & 0.101 & 0.030 \\
\hline CD @ 5\% & 1.554 & 1.955 & 2.177 & 0.391 & 0.340 & 0.292 & 0.359 & 0.246 & 2.219 & 0.288 & 0.086 \\
\hline $\mathrm{CV} \%$ & 4.717 & 4.711 & 4.533 & 0.749 & 2.051 & 0.622 & 0.216 & 10.006 & 0.436 & 6.090 & 6.090 \\
\hline
\end{tabular}

*Pooled analysis; In each column, means followed by the same letter are not significantly different at $\mathrm{p}=0.05$ according to Duncan's Multiple Range Test. 
Plate.1 Different biotic defense inducers used for the management of CMV disease T1- Red seaweed (Kappaphycus alvarezii (Doty) Doty ex P.C. Silva) extract (LBD 3); T2Synthetic nucleoside (SEVI); T3- Extract of Mirabilis jalapa L. (Sanjemallige); T4- Extract of Bougainvillea spectabilis Willd. (Paper flower); T5- Fungal bio-control agent (Trichoderma harzianum); T6- Bacterial bio-control agent (Pseudomonas fluorescens); T7- Chitosan; T8Virex-H; T9- Dimethoate 30\% EC

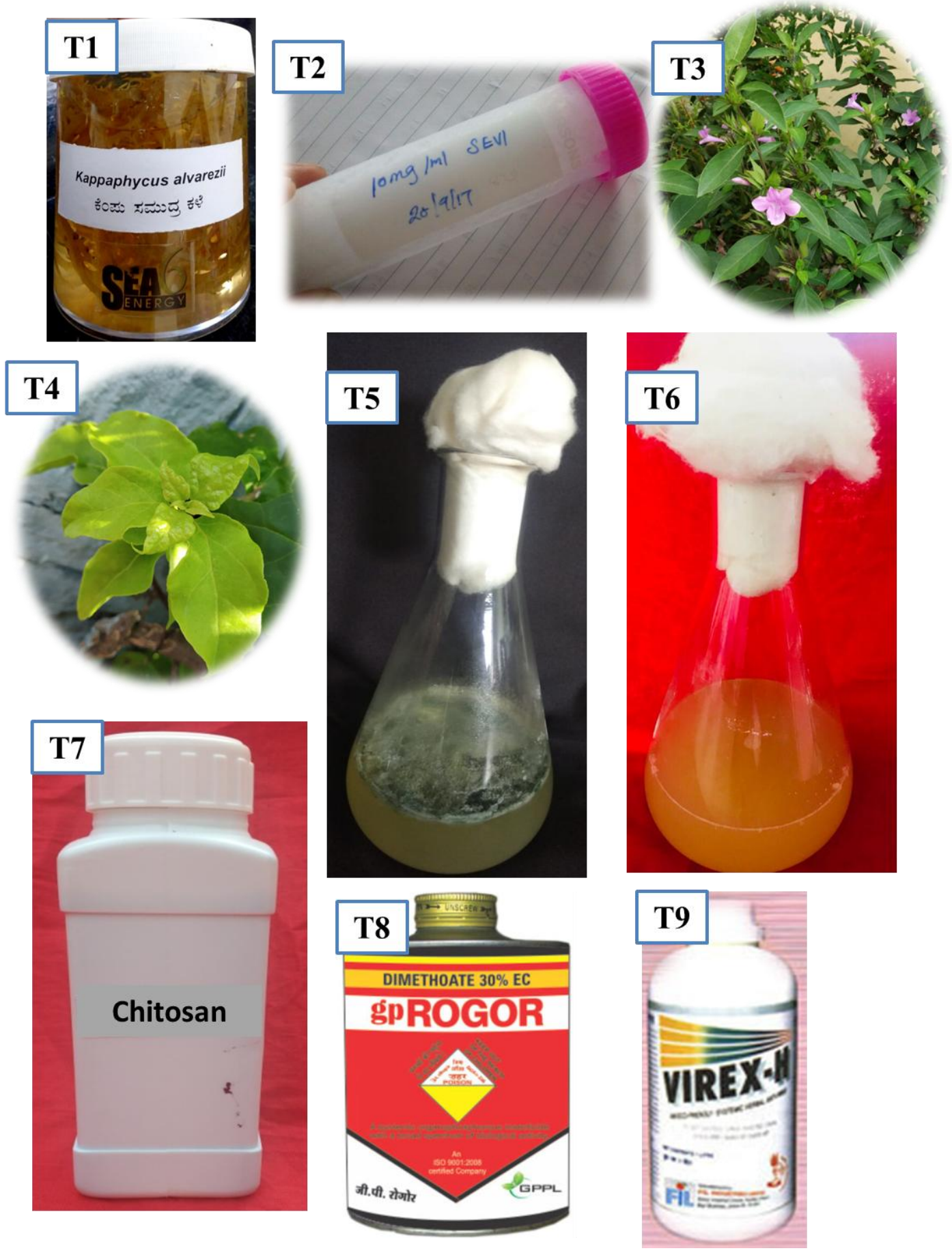


Plate.2 Field view for the management of CMV disease in chilli using different biotic inducers at MRS, Hebbal, Bengaluru

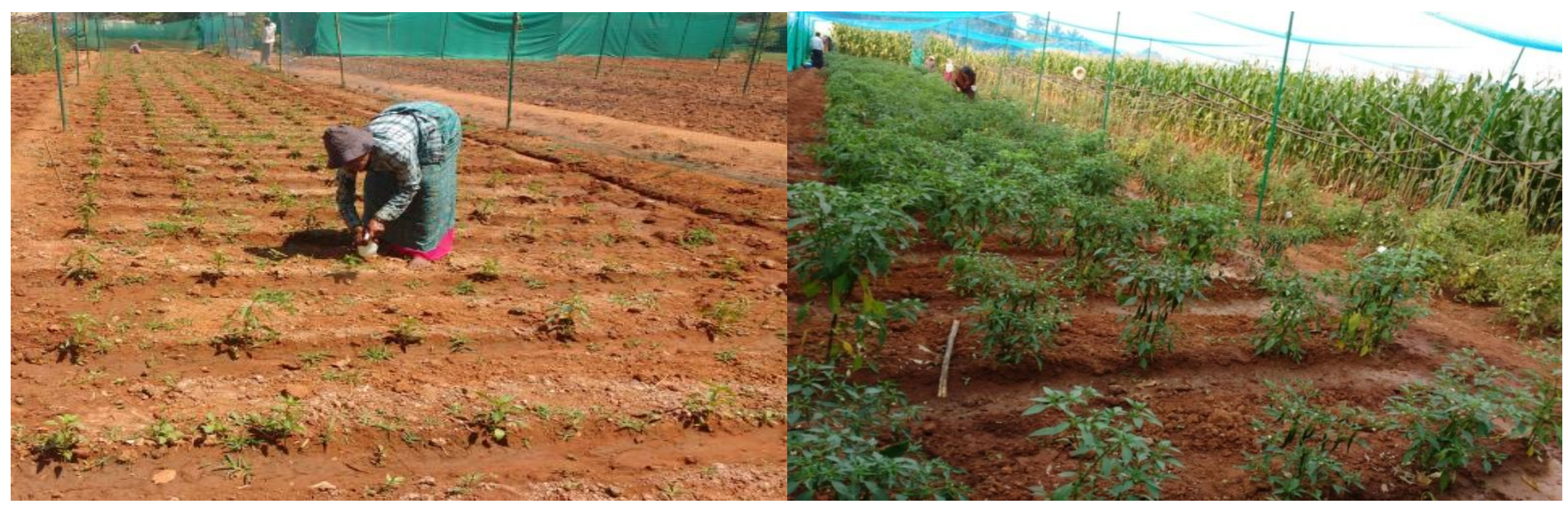

Plate.3 Symptoms of CMV on naturally infected chilli plants

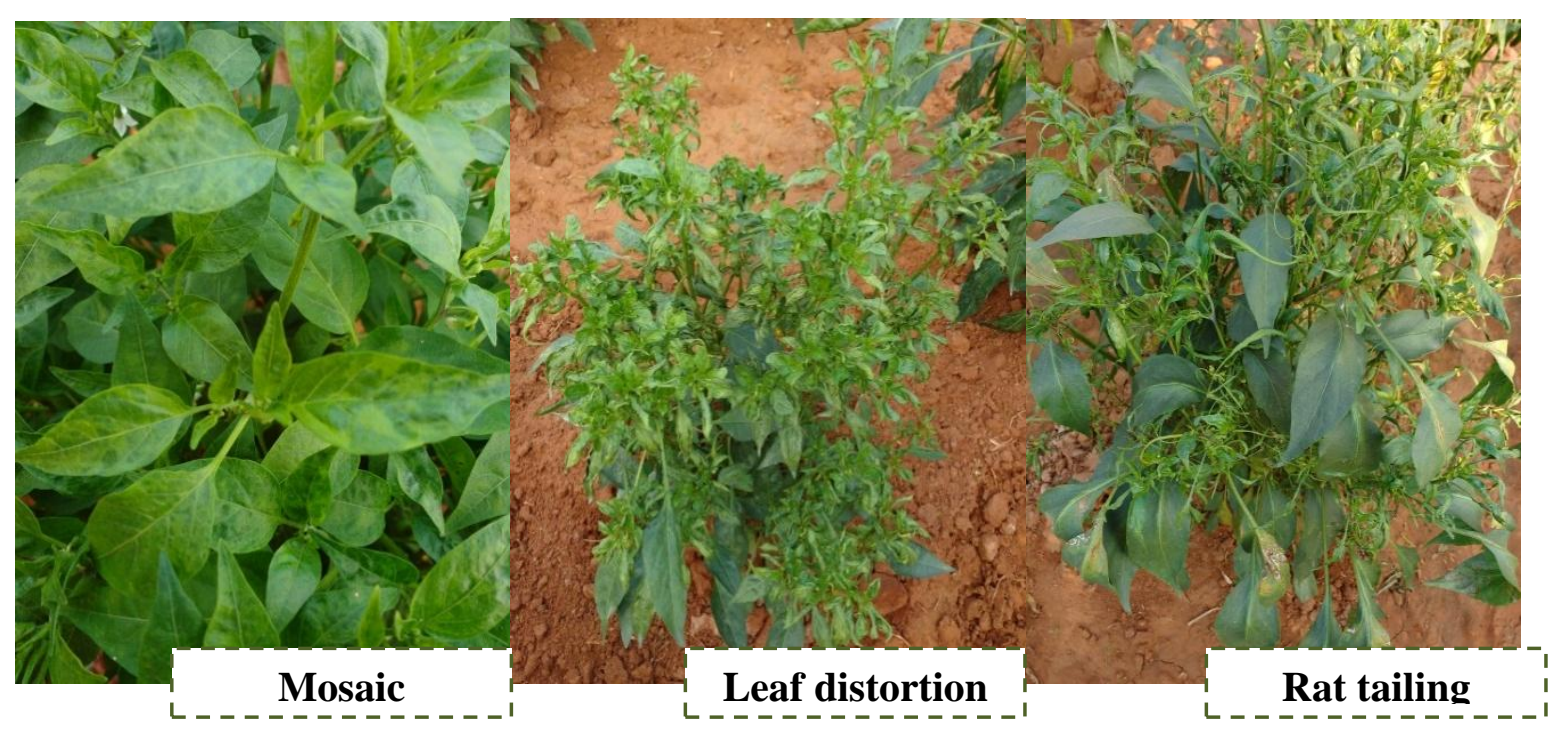


Fig.1 Effect of biotic inducers on the incidence of the CMV disease during Kharif 2016-17 \& 2017-18 under field conditions

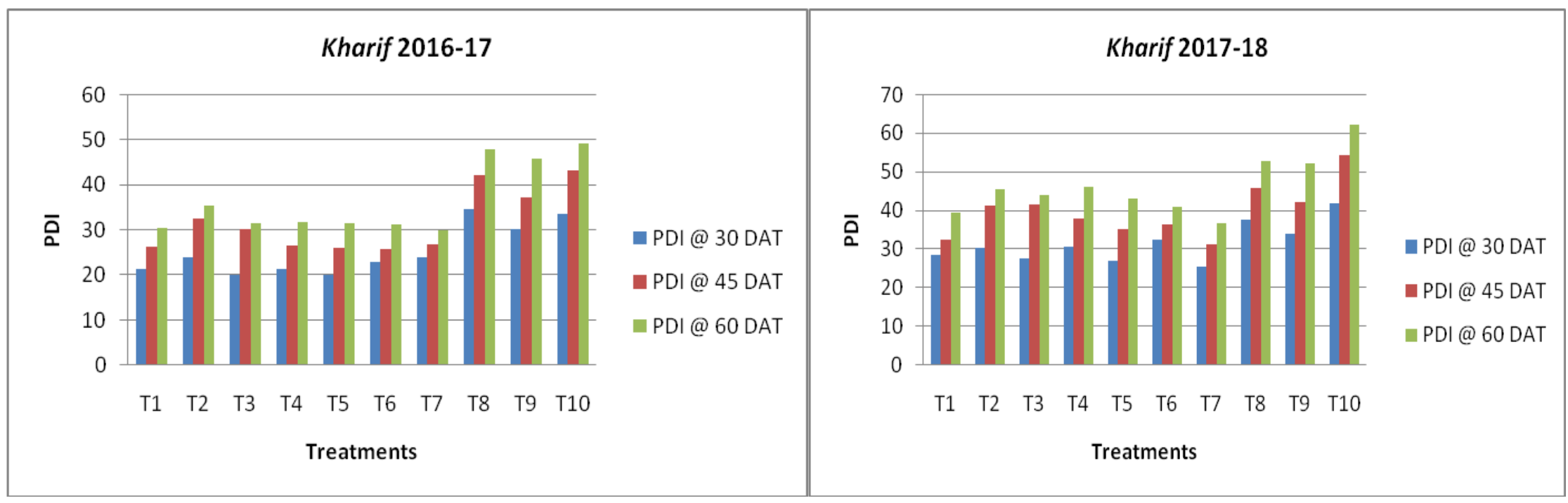

Fig.2 Effect of biotic inducers on growth parameters of chilli during Kharif 2016-17 \& 2017-18 under field conditions

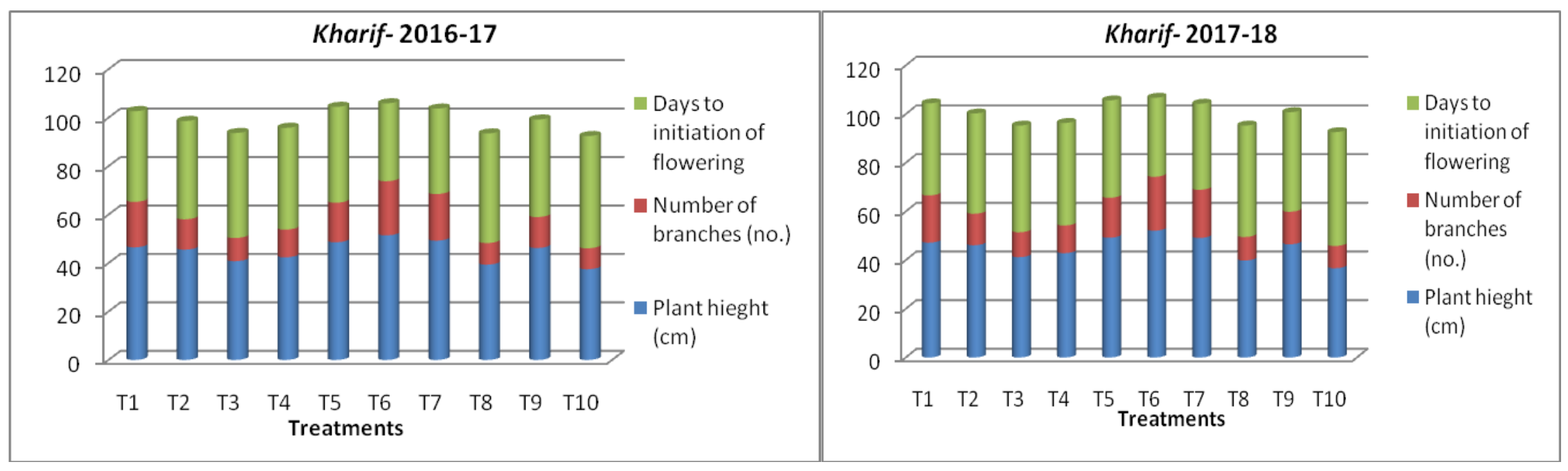


Similar results were also reported by Noiket et al., (2014), Agbodjato et al., (2015), Jelinet al., (2013), Aleksandrowicz-Trzcinska et al., (2015) respectively in tomato, maize and scots pine sprayed with chitosan and PGPR. The highest plant length, fresh and dry weights were obtained in tomato plants grown from transplants treated with chitosan (Nawar, 2005). Application of chitosan (5\%) had the superior effect (El-Tanahy et al., 2012) on all measured vegetative parameters of cowpea plants. Foliar application of chitosan increased common bean plant growth as compared to chitosan untreated plants (Abu-Muriefah, 2013). Besides, chitosan also promoted the growth and yield of tomato (Ibrahim et al., 2015).

In the present study enhanced growth was observed in the plants treated with $P$. fluorescens. Application of PGPR has promoted plant growth by facilitating resource acquisition, modulating plant hormone levels and decreasing the inhibitory effects of various pathogens as biocontrol agents (Glick, 2012).

Plant yield promotion by different biotic inducers

Analysis on yield components showed that, number of fruits per plant, individual fruit weight, fresh weight and dry weight of chilli was influenced by several treatments (Table 4). Application of chitosan (0.1\%), $K$. alvarezii (0.4\%) and P. fluorescens (0.6\%) significantly improved plant yield compared to control under field condition during Kharif2016-17 and Kharif2017-18.

Data from the table 4 elucidated that, chitosan treated chilli plants was found effective significantly by producing highest yield of 1.47 and 1.29 t.ha $^{-1}$ during Kharif2016-17 and Kharif 2017-18 compared to untreated control (1.19 and 0.97 t.ha $\left.^{-1}\right)$. The similar trend was also observed in the plants sprayed with $K$. alvarezii and $P$. fluorescens by recording dry weight of 1.42 and 1.24 t.ha $^{-1}$ and 1.40 and 1.19 t.ha $^{-1}$ respectively. Chitosan spray significantly increased number of fruits per plant (192.70 and 193.04), individual fruit weight (2.56 and $2.62 \mathrm{~g})$, fresh weight (4.89 and 4.89 t.ha $^{-1}$ ), dry weight (1.47 and 1.47) of chilli compared with the control respectively during Kharif 2016-17 and Kharif2017-18.

These results are in agreement with the report made by El- Mougy et al., (2006), chitosan treated tomato plants showed increased yield by 66.7 per cent compared with untreated plants. Additionally, El-Tanahy et al., (2012) pointed out that the best yield of cowpea plants were obtained by using chitosan (1213.89 g.plot $\left.{ }^{-1}\right)$. Foliar application of tomatoes with extract of brown seaweed, Ascophyllum nodosum (0.5\%) (ANE) reduced disease incidence of Alternaria solani and Xanthomonas campestris pv vesicatoria upto 63 and 44 per cent respectively with increased fruit yield upto 42 per cent compared to controls (Ali et al., 2016).

Similarly, Venkatesh (2016) found increased mean yield of gherkins by 13.33 t.ha ${ }^{-1}$ and 12.17 t.ha $^{-1}$ in gherkin plants sprayed with seaweed extracts $K$. alvarezii-1 $(0.4 \%)$ and Halymenia durvillae (1\%) respectively in Kharif and rabi 2016 field experiments. Mishra et al., (2014) reported that, the tomato plants treated with the chitosan based formulation of Pseudomonas sp.(206(4) +B$15+\mathrm{JK}-16)$ recorded the highest activity of ISR against ToLCV and recorded maximum plant height $(52.5 \pm 1.38 \mathrm{~cm})$, total biomass $\left(0.043 \pm 0.041 \mathrm{~kg} \cdot\right.$ plant $\left.^{-1}\right)$, chlorophyll content (35.2 \pm 1.02 SPAD), fruit number $(26.6 \pm 0.81)$ and yield $\left(1.77 \pm 0.0 .07 \mathrm{~kg} . \mathrm{plant}^{-1}\right)$ over the diseased control $\left(0.45 \pm 0.01 \mathrm{~kg}\right.$.plant $\left.{ }^{-1}\right)$. The efficacy of $K$. Alvarezii-1(0.4\%) against papaya ringspot virus (PRSV) was tested under field condition by Vijayalakshmi and 
Nagaraju (2017), where K.alvarezii-1has recorded 71.66 per cent disease control with the yield of $41.87 \mathrm{~kg}$.plant ${ }^{-1}$ compared to untreated control which showed 100 per cent disease incidence.

Pooled analysis of data obtained from both the seasons Kharif2016-17 and Kharif201718 also confirmed the effectiveness of chitosan (0.1\%), K. Alvarezii (0.4\%), Synthetic nucleoside $(0.25 \%)$ and $P$. fluorescens $(0.6 \%)$ in reducing the CMV disease incidence by increasing the growth and yield parameters (Table 5). Among them, Chitosan and $K$. Alvarezii showed significantly increased yield of about 1.38 and 1.33 t.ha $^{-1}$ respectively followed by $P$. fluorescens (1.30 t.ha $\left.{ }^{-1}\right)$ and Synthetic nucleoside (1.28 t.ha $\left.{ }^{-1}\right)$.

It is concluded in the present study, the increased chilli yield was observed due to reduction in CMV disease incidence with enhanced growth influenced by foliar application of Chitosan, $K$. alvarezii and $P$. fluorescens. Therefore it is possible to recommend above treatments to manage CMV infection effectively under field conditions with enhanced growth and yield of chilli.

\section{Acknowledgement}

I duly acknowledge to Department of Plant Pathology, UAS, GKVK and Main Research Station (MRS), Hebbal, Bengaluru for providing experimental field facility and to UGC, Govt. of India for the financial assistance during Ph.D. (National fellowship for OBC students NF-OBC).

\section{References}

Abu-Muriefah, S.S., 2013, Effect of chitosan on common bean (Phaseolus vulgaris L.) plants grown under water stress conditions. Int. Res. J. Agril. Sci. Soil Sci., 3(6): 192-199.

Agbodjato, N.A., Noumavo, P.A., Adjanohoun, A., Dagbenonbakin, G., Atta, M., Rodriguez, A.F., Pons, B.M. and Baba-Moussa, L., 2015, Response of maize (Zea mays L.) crop to biofertilization with plant growth promoting Rhizobacteria and chitosan under field conditions. J. Exp. Biol. Agric. Sci., 3(6): 566-574.

Agranovsky, A.A., 1993, Virus diseases of pepper (Capsicum annuumL.) in Ethiopia. J. Phytopathol., 138: 89-97.

Aleksandrowicz-Trzcinska, M., A. Bogusiewicz, M. Szkop and S. Drozdowski, 2015, Effect of chitosan on disease control and growth of Scots Pine (Pinussylvestris L.) in a forest nursery. Forests, 6: 3165-3176.

Ali, N., Ramkissoon, A., Ramsubhag, A. and Jayaraj, J., 2016, Ascophyllum extract application causes reduction of disease levels in field tomatoes grown in a tropical environment. Crop Protect., 83: 67-75.

Astuti, U.P., Wahyuni, T and Honorita, B., 2013, Technical guidelines for the manufacture of vegetable pesticides. Agricultural Technology Assessment Center (BPTP) Bengkulu.Bengkulu.75 p.

Bhadramurthy, V., George, A., Bhat, A.I. and Shiva, K.N., 2009, Coat protein gene sequence studies suggest that Cucumber mosaic virus infecting paprika (Capsicum annuum L.) in India belongs to subgroup IB. Archives Phytopathol. Pl. Prot., 42(9): 857-863.

Biswas, K., Hallan V., Zaidi, A.A., Pandey, P.K., 2013, Molecular evidence of Cucumber mosaic virus subgroup II infecting Capsicum annuum L. in the Western region of India. Int. J. Curr. Discov. Innov., 2: 97-105

Boller, T. and Felix, G., 2009. A renaissance 
of elicitors: perception of microbeassociated molecular patterns and danger signals by pattern-recognition receptors. Annual review Pl. Biol., 60:379-406.

Campbell, C. L. and Madden, L. V., 1990, Introduction to plant disease epidemiology. Wiley, New York, USA.

Chen, B. and Francki, R. I. B., 1990, Cucumovirus transmission by the aphid Myzuspersicae is determined solely by the coat protein. J. Gen Virol.,71:939944.

Compant, S., C. Clement and A. Sessitsch, 2010, Plant Growth-promoting bacteria in the rhizo-and endosphere of plants: Their role, colonization, mechanisms involved and prospects for utilization. Soil Biol. Biochem., 42: 669-678.

Daayf, F., Bel-Rhlid, R., Belanger, R.R., 1997, Methyl easter of P- coumaric acid: A phytoalexin like compound from long English cucumber leaves. $J$. Chem. Eco. 23: 1517-1526.

Deni-Firmansyah, Widodo and Sri Hendrastuti Hidayat, 2017, Chitosan and plant growth promoting rhizobacteria application to control Squash mosaic virus on cucumber plants. Asian J. Pl. Pathol., 11: 148155.

El-Mougy, N.S., N.G. El-Gamal, Y.O. Fotouh and F. Abd-El-Kareem, 2006, Evaluation of Different Application Methods of Chitin and Chitosan for Controlling Tomato Root Rot Disease under Greenhouse and Field Conditions. Res. J. Agric. and Biol. Sci., 2(5): 190195.

El-Tanahy, A.M., A.R. Mahmoud, M.M. Abde-Mouty and A.H. Ali, 2012, Effect of chitosan doses and nitrogen sources on the growth, yield and seed quality of cowpea. Aust. J. Basic and Appl. Sci., 6(4): 115-121.

Glick, B.R., 2012, Plant growth-promoting bacteria: mechanisms and applications. Scientifica.

Green, S. K. and Kim, J. S., 1991, Characterization and control of pepper viruses: A literature review. AVRDC Tech. Bull., 18: 1-60.

Grube, R.C., Zhang, Y., Murphy, J.F., LoaizaFigueroa, F., Lackney, V.K., Provvidenti, R. and Jahn, M.K., 2000.New source of resistance to Cucumber mosaic virus in Capsicum frutescens. Pl. Dis., 84:885-891.

Hadrami, E.A., L.R. Adam, I. El-Hadrami and F. Daayf, 2010, Chitosan in plant protection. Marine Drugs, 8: 968-987.

Harish, S., 2005, Molecular biology and diagnosis of banana bunchy top virus and its management through induced systemic resistance. Ph.D. Thesis, Tamil Nadu Agricultural University Coimbatore (India).

Ibrahim, E.A. and W.A. Ramadan, 2015, Effect of zinc foliar spray alone and combined with humic acid or/and chitosan on growth, nutrient elements content and yield of dry bean (Phaseolus vulgaris L.) plants sown at different dates. Scientia Horticulturae, 184: 101-105

IHD (Indian horticultural Database), 2015, National Horticulture Board, Ministry of Agriculture, Government of India 85, Institutional Area, Sector-18, Gurgaon122 015, INDIA.

Jelin, J., T.A. Selvakumar and M.S. Dhanarajan, 2013, Phytological analysis for designing a microbial consortium to enhance plant growth. Int. J. Chem Tech Res., 5: 1370-1375.

Jones, J.W., Dayan, E., Allen, L.H., Van Keulen, H. and Challa, H., 1991, A dynamic tomato growth and yield model (TOMGRO). Transactions of the ASAE, 34: 663-672.

Kandan, A., Radjacommare, R., Ramiah, M., Ramanathan, A., Samiyappan, R., 2003, 
PGPR induced systemic resistance in cowpea against tomato spotted wilt virus by activating defense against tomato spotted wilt virus by activating defense related enzymes and compound. In: Proceedings of the Sixth International Workshop on Plant Growth Promoting Rhizobacteria $E d$ YR Sarma IISR Publishers Calicut, 480486.

Macho, A.P. and Zipfel, C., 2014. Plant PRRs and the activation of innate immune signaling. Molecular cell, 54(2):263272.

Madhusudhan, K.N., Nalini, M.S., Prakash, H.S. and Shetty, H.S., 2005, Effect of inducers against tobamovirus infection in tomato and bell pepper. Int. J. Bot., 1: 59-61.

Maurhofer, M., Hase, C., Meuwly, P., Metraux, J.P. and Defago, G., 1994. Induction of systemic resistance of tobacco to tobacco necrosis virus by the root-colonizing Pseudomonas fluorescens strain CHA0: influence of the gacA gene and of pyoverdine production. Phytopathol., 84: 139-146.

Mishra, S., Jagadeesh, K.S., Krishnaraj, P.U. and Prem, S., 2014, Biocontrol of tomato leaf curl virus (ToLCV) in tomato with chitosan supplemented formulations of Pseudomonas sp. under field conditions. Australian J. Crop Sci., 8(3): p.347.

Murphy, J. F., 2006, Applied aspects of induced resistance to plant virus infection. In: Natural Resistance Mechanisms of Plants to Viruses. G. Loebenstein and J. P. Carr, eds. Springer, The Netherlands. pp 1-11

Murphy, J.F., Zehnder, G.W., Schuster, D.J., Sikora, E.J., Polstan, J.E. and Kloepper, J.W., 2000, Plant growth-promoting rhizobacteria mediated protection in tomato against tomato mottle virus. $\mathrm{Pl}$. Dis., 84(7): 779-784.
Myti, S., Shabbir, A.K., Akhter, S., Uddin, A., Kamruzzaman, M., Faruq, M.O. and Biswas, G.C., 2014, Identification of the most prevalent and spatially disperse virus on chilli at Northern and Eastern part of Bangladesh. Int. J. Biosci., 5: 4049.

Nawar, L.S., 2005, Chitosan and three Trichoderma spp. to control fusarium crown and root rot of tomato in jeddah, Kingdom Saudi Arabia. Egypt. J. Phytopathol, 33(1): 45-58.

Neergaard, P., 1977, Seed Pathology. Macmillan, London. I, II:1187.

Noiket, N., T. Boonthip and K. Riangwong, 2014, Evaluation of potential for chitosan to control TYLCV disease and promote the growth of Sridathip 3 tomato. Proceedings of the 26th Annual Meeting of the Thai Society for Biotechnology and International Conference, November 26-29, 2014, Chiang Rai, Thailand, pp: 252-259.

Ong, C.A., Varghese, G. and Ting, W.P., 1979, Aetiological in- vestigations on a veinal mottle virus of chilli (Capsicum annuum L.) newly recorded from Peninsular Malaysia. MARDI Res. Bulletin, 7: 78-88.

Palukaitis, P. and Garcia Arenal, F., 2003, Cucumoviruses.Adv. in Virus Res., 62: 241-323.

Podile AR, Laxmi VDV (1998) Seed bacterization with Bacillus subtilis AF1 increases phenylalanine ammonia-lyase and reduces the incidence of fusarial wilt in pigeon pea. $\mathbf{J}$ Phytopathol 146:255-259.

Pushpa R.N., Shantamma, Anil Pappachan, Manjunath B., BhoseSumit, Sawan Kumar, Rangaswamy K.T., Girish T.R. and Nagaraju N., 2018, Molecular Characterization, Epidemiology and Management of the Papaya ringspot virus (PRSV) in Papaya under Southern Indian Conditions. Int. J. Agri. Sci., 
10(2): 5029-5038.

Rashid, M.H., Khalequzzaman, K.M., Alam, M.S., Uddin, S.A. and Green, S.K., 2007.Screening of different sweet pepper lines against cucumber mosaic virus and chiliveinal mottle virus.Int. J. Sustain. Crop Prod. 2(3):1-4.

Sheoran, O.P., Tonk, D.S., Kaushik, L.S., Hasija, R.C and Pannu, R.S., 1998, Statistical Software Package for Agricultural Research Workers. Recent Advances in information theory, Statistics \& Computer Applications by D.S. Hooda \& R.C. Hasija Department of Mathematics Statistics, CCS HAU, Hisar: 139-143.

Thippeswamy, P., 2010, Evaluation of botanicals and panchagavya against selected fungal diseases of paddy under organic farming system. M.Sc. (Agri.) Thesis, Uni. Agric. Sci., Bangalore.

Van -Loon, L.C., 1997, Induced resistance in plants and the role of pathogenesis related proteins. Eur. J. Pl. Pathol., 103: 753-765.
Venkatesh, H.L., 2016, Host-plant resistance against cucumber mosaic virus and its organic management in gherkins (Cucumis sativus L.). M.Sc. (Agri.) Thesis, Univ. Agri. Sci. Bangalore.

Vera, J., Castro, J., Gonzalez, A., Barrientos, H., Matsuhiro, B., Arce, P., Zuniga G. and Moenne, A., 2011, Molecular Pl. Pathol., 12(5): 437-447.

Vijayalakshmi, G. and Nagaraju, N., 2017, Non-chemical management of Papaya ring spot virus in papaya (Carica papaya L.). Mysore J. Agricl. Sci., 51(3): 625-630.

Villalon, B., 1981, Breeding peppers to resist virus diseases. $\mathrm{Pl}$. Dis., 65(7):557-561.

Vincent, J. M., 1927, Distortion of fungal hyphae in the certain inhibitors. Nature, 159: 850.

Vishwanath, K. and Kolte, S. J., 1997, Variability in Alternaria brassicae: Response to host genotypes, toxin production and fungicides. Indian Phytopathol.,50: 373- 381.

\section{How to cite this article:}

Kavyashri, V.V. and Nagaraju, N. 2019. Techniques for Determination of Vitamin B6, Vitamin C and Variability in Areca Nut. Int.J.Curr.Microbiol.App.Sci. 8(01): 297-313. doi: https://doi.org/10.20546/ijcmas.2019.801.032 